ISBN 82-553-0582-3

Pure Mathematics
No 5

June, 1985

\title{
ABSTRACT HIERARCHIES AND DEGREES
}

by

I.I. Ivanov

PREPRINT SERIES - Matematisk institutt, Universitetet i Oslo 


\title{
ABSTRACT HIERARCHIES AND DEGREES
}

\author{
by \\ I.I. Ivanov
}

The aim of this report is to enrich the axiomaticalgebraic approach to Recursion Theory developed in [1-4] by an analog to the classical arithmetical hierarchy and also an abstract notion of degree. The presented results are rather initial ard elementary; indeed, the main problem was the very choice of true abstract concepts. 
0. Preliminaries.

In this section we briefly (and somewhat chaotically) remind certain definitions and facts needed for the present considerations. Followed is the "lightface" version of the general theory.

Assume that an iterative operative space $S=(F, I, \Pi, I, R)$ is given, i.e. $(F, 0, \leqq)$ is a partially ordered semigroup with a unit $I, I \neq R \in F, \Pi: F^{2} \rightarrow F$ is monotonous s.t. $(\varphi, \psi) \chi=(\varphi x, \psi \chi)$, $I(\varphi, \psi)=\varphi, R(\varphi, \psi)=\psi \quad($ writing $(\varphi, \psi)$ for $\Pi(\varphi, \psi))$, and the operations $\langle>=\lambda \varphi \cdot \mu \theta \cdot(\varphi I, \theta R),[]=\lambda \varphi \cdot \mu \theta \cdot(I, \varphi \theta)$ exist and meet a $\mu$-induction axiom.

An element $\varphi$ is recursive in a subset $B$ of $F$ iff it can be obtained from $L, R$ and members of $B$ by consecutive applications of the basic operations $0, \pi,\langle\rangle,[]$. It is the case that $O=R[R] \leqq \varphi$ and $O \varphi=0$ for all $\varphi, \bar{n}\langle\varphi\rangle=\varphi \bar{n}$ and $\bar{n}[I]=I$ for all $n, \bar{n}$ standing for $L R^{n}$. There are recursive (in $\varnothing)$ elements $C, D$ s.t. $\bar{n} C=(\bar{n} I, \bar{n} R)$ and $\bar{n} D=\bar{n} \bar{n}$ for all $n$. Whenever $\bar{n} \varphi \leqq \bar{n} \psi$ for all $n$, then $\langle I\rangle \varphi \leqq\langle I\rangle \psi$; in this work we shall consider spaces with $\langle I\rangle=I$, hence the latter inequality implies $\varphi \leqq \psi$.

The notion of a mapping over $F$ recursive in $B$ is introduced via parametrization. There exists a universal unary recursive mapping $\Sigma$, which means that whenever $\Gamma$ is a unary recursive mapping, then $\Gamma=\lambda \theta \cdot \bar{m} \Sigma(\theta)$ for a certain $m$. It follows in particular that for all $\psi$ the element $\Sigma(\psi)$ is recursive in $\psi$ and universal for all such elements, i.e. whenever $\varphi$ is recursive in $\psi$, then $\varphi=\bar{m} \Sigma(\psi)$ for a certain $m$. 
Theorem 1.1. Let $S$ be the space of example 2 [2], i.e. $F=\{\varphi / \varphi: \omega-\rightarrow \omega\}, \varphi \leqq \psi$ iff $\varphi \subseteq \psi, \varphi \psi=\lambda s \cdot \psi(\varphi(s))$, $(\varphi, \psi)(2 s)=\varphi(s),(\varphi, \psi)(2 s+1)=\psi(s), I=\lambda s \cdot s, I=\lambda s \cdot 2 s$ and $R=\lambda s \cdot 2 s+1$. (Cf. [2] for explicit characterizations of the operations $\langle>,[]$.$) Take by definition (\varphi \vee \psi)(s)=\varphi(s)$, if $\varphi(s) \psi$, and $(\varphi \vee \psi)(s)=\psi(s)$ otherwise. Then the axioms of adjunction operation are valid.

The verification is straightforward and not adduced. Some general properties of adjunction follow, formulated in terms of its techincally more convenient modification +, where $\varphi^{+}=\varphi L \vee R$. In the above example $\varphi^{+}(s)=2 \varphi(s)$, if $\varphi(s) \downarrow$, and $\varphi^{+}(s)=2 s+1$ otherwise.

Lemma 1.2. $\varphi \vee \psi=\varphi^{+}(I, \psi)$.

Proof: It follows by the axioms that

$$
(\varphi \vee \psi) I=\varphi I \vee \psi L=\varphi^{+}(I, \psi I)=\varphi^{+}(I, \psi) I \text {. }
$$

Multiplying by $(I, I)$, one gets the desired equality.

Lemma 1.3. $I^{+}=I, O^{+}=R$.

Proof: $I^{+}=I V R=I, O^{+}=$OVR $=R$.

Lemma 1.3 shows in particular that + is not monotonous since $0 \leqq I$ but $\mathrm{O}^{+} \$ \mathrm{I}^{+}$. Adjunction is not monotonous on its first argument and monotonous on its second argument. 
Lemma 1.10. $(\varphi, \psi)^{+}=\left(\varphi^{+}(I, I R), \psi^{+}\left(I, R^{2}\right)\right)$.

Proof: The equality $\langle I\rangle=I$ implies $(I, R)=I$, hence

$$
\begin{aligned}
(\varphi, \psi)^{+} & =(I, R)(\varphi, \psi)^{+}=\left(I(\varphi, \psi)+, R(\varphi, \psi)^{+}\right) \\
& =\left((I(\varphi, \psi))+(I, I R),(R(\varphi, \psi))+\left(I, R^{2}\right)\right) \\
& =\left(\varphi^{+}(I, I R), \psi^{+}\left(I, R^{2}\right)\right) .
\end{aligned}
$$

An element $\varphi$ is total iff $\varphi^{+}=\varphi \mathrm{I}$. Write Tot for the set of all total elements in $F$.

Lemma 1.11. $0 \notin$ Tot, $I \in$ Tot.

Proof:

$$
O^{+}=R \neq O=O L,
$$

while $I^{+}=I$.

Lemma 1.12. $F^{+} \subseteq$ Tot.

Follows by 1.4 .

Lemma 1.13. Whenever $\varphi \in$ Tot, then $\alpha \varphi, \varphi \alpha \in$ Tot for $\alpha=I, R$. Proof:

$$
\begin{aligned}
(\varphi \alpha)^{+} & =\varphi^{+}(\alpha I, R)=\varphi I(\alpha I, R)=\varphi \alpha I, \\
(\alpha \varphi)^{+} & =(\alpha \varphi)^{+}(I, \alpha R)(I,(R, R))=\alpha \varphi^{+}(I,(R, R)) \\
& =\alpha \varphi L(I,(R, R))=\alpha \varphi I .
\end{aligned}
$$

Lemma 1.14. If $\bar{n} \varphi \in$ Tot for all $n$, then $\varphi \in$ Tot. Proof: It follows that

$$
\bar{n} \varphi^{+}=(\bar{n} \varphi)+(I, \bar{n} R)=\bar{n} \varphi L(I, \bar{n} R)=\bar{n} \varphi I
$$

for all $n$, hence $\varphi^{+}=\varphi I$. 
Some elementary properties.

Theorem 2.1 (Hierarchy Theorem) $\cdot \Delta_{n} \subseteq \Sigma_{n} \subseteq \Delta_{n+1} \cdot$

The former inclusion follows by 1.5 , the latter is immediate.

Lemma 2.2. $\Delta_{1}=\Delta_{0}, \Sigma_{1}=\{\varphi / \varphi$ is recursive $\}$.

Follows by the corresponding definitions.

Lemma 2.3. $\Delta_{n}^{+} \Delta_{n} v \Delta_{n} \subseteq \Delta_{n}$.

Follows by $1.4,1.6$.

Lemma 2.4. $\Delta_{n}=+^{-1} \cdot\left(\Sigma_{n}\right)$.

Follows by the definitions.

Lemma 2.5. $\quad \Sigma_{n} \cap$ Tot $\subseteq \Delta_{n}$.

Proof: Whenever $\varphi \in \Sigma_{n} \cap$ Tot, then $\varphi^{+}=\varphi I \in \Sigma_{n}$, hence $\varphi \in \Delta_{n}$ by 2.4 .

Theorem 2.6 (Enumeration Theorem).

There is an element $\sigma_{n+1} \in \Sigma_{n+1}$ universal for $\Sigma_{n+1}$.

Proof: The element $\sigma_{1}=\Sigma(I) \in \Sigma_{1}$ is universal for $\Sigma_{1}$.

Suppose that $\sigma_{n+1} \in \Sigma_{n+1}$ is universal for $\Sigma_{n+1}$, i.e.

$\Sigma_{n+1} \subseteq\left\{\bar{m} \sigma_{n+1} / m \in \omega\right\}$. Then the definition of $\Sigma_{n+1}$ implies ,$\Sigma_{n+1}=\left\{\bar{m}_{n+1} / \mathrm{m} \in \omega\right\}$, hence

$$
\Sigma_{n+1}^{+}=\left\{\left(\bar{m} \sigma_{n+1}\right)^{+} / m \in \omega\right\}=\left\{\bar{m} \sigma_{n+1}^{+}(I,[I] R) / m \in \omega\right\} \text {. }
$$

One gets

$$
\begin{aligned}
\Sigma_{n+2} & =\left\{\varphi / \varphi \text { is recursive in } \Sigma_{n+1}^{+}\right\} \\
& =\left\{\varphi / \varphi \text { is recursive in } \sigma_{n+1}^{+}\right\},
\end{aligned}
$$

hence the element $\sigma_{n+2}=\Sigma\left(\sigma_{n+1}^{+}\right) \in \Sigma_{n+2}$ is universal for $\Sigma_{n+2} \cdot$ The proof is completed. 
Proof: Whenever $f(s) \downarrow$, then

$$
\begin{aligned}
& \bar{s} \psi=(\bar{s} \varphi)+(L, \bar{s} R)\left(\rho_{1}, \rho_{2}\right)=\overline{\overline{f(s)}}+\left(\rho_{1}, \bar{s} \rho_{2}\right) \\
& =\overline{f(s)} I\left(\rho_{1}, \bar{s} \rho_{2}\right)=\overline{f(s)} \rho_{1}=\overline{2 f(s)}=\overline{f^{+}(s)} \text {. }
\end{aligned}
$$

Otherwise

$$
\begin{aligned}
\bar{s} \psi & =(\bar{s} \varphi)^{+}\left(\rho_{1}, \bar{s} \rho_{2}\right)=o^{+}\left(\rho_{1}, \overline{2 s+1}\right) \\
& =R\left(\rho_{1}, \overline{2 s+1}\right)=\overline{2 s+1}=\overline{f^{+}(s)} .
\end{aligned}
$$

Therefore, $\psi$ represents $\mathrm{f}^{+}$.

Lemma 2.11. If $\psi$ represents $f^{+}$, then $\varphi=\psi \rho(I, 0)$ represents $f$ and $\varphi^{+}=D\langle\psi \rho\rangle C([I] I, R)$, where $\rho$ is a recursive element s.t. $\overline{2 s} \dot{s}=\bar{s} I$ and $\overline{2 s+1} \rho=R$.

Proof: Whenever $f(s) \downarrow$, then

$$
\begin{aligned}
\bar{s} \varphi & =\bar{s} \psi \rho(I, 0)=\overline{f^{+}(s)} \rho(I, 0) \\
& =\overline{2 f(s)} \rho(I, 0)=\overline{f(s)} I(I, 0)=\overline{E(s)} .
\end{aligned}
$$

Otherwise

$$
\bar{s} \varphi=\overline{E^{+}(s)} \rho(I, 0)=\overline{2 s+1} \rho(I, O)=R(I, O)=0,
$$

hence $\varphi$ represents $f$. It is the case that

$$
\begin{aligned}
s D & <\psi \rho>C([I] L, R)=\bar{s} \bar{s}\langle\psi \rho>C([I] I, R) \\
& =\bar{s} \psi \rho \bar{s} C([I] L, R)=\overline{E^{+}(s)} \rho(I, \bar{s} R) \\
& =(\bar{s} \varphi)^{+}(I, \bar{s} R)=\bar{s} \varphi^{+}
\end{aligned}
$$

for all $s$, hence $\varphi^{+}=D\langle\psi \rho>C([I] L, R)$. Thereby the proof is completed. 
Theorem 2.13.

Let $S$ be the space of 1.1 and $z=\lambda s .(2 s+1) \mathrm{sgs}$. Then $\Sigma_{n}^{0} \cap F=\Sigma_{n}(Z)$ and $\Delta_{n}^{0} \cap F=\Delta_{n}(z)$.

Proof: It is known [2] that $\varphi$ is $\mu$-recursive in $\psi$ iff $\varphi$ is recursive in $\mathrm{Z}, \psi$; notice also that $\mathrm{Z} \in$ Tot.

The equality $\Sigma_{0}^{0} \cap F=\Sigma_{0}(z)$ follows by 2.9 .

Suppose that $\Sigma_{n}^{0} \cap F=\Sigma_{n}(z)$. By $2.9 \varphi \in \Sigma_{n+1}^{0}$ iff $\varphi$ is $\mu$-recursive in $\psi^{+}$for a certain $\psi \in \Sigma_{n}^{0}$. The latter is equivalent to $\varphi$ being recursive in $Z_{,} \psi^{+}$for a certain $\psi \in \Sigma_{n}(z)$, which in turn is equivalent to $\varphi \in \Sigma_{n+1}(z)$. Finally,

$$
\Delta_{n}^{0} \cap F=+^{-1}\left(\Sigma_{n}^{0} \cap F\right)=+^{-1}\left(\Sigma_{n}(Z)\right)=\Delta_{n}(Z)
$$

completes the proof.

Besides relativization, projective hierarchies can be modified in another aspect by replacing recursiveness by other abstract notions of computability s.a. t-recursiveness and $B^{\prime}$-recursiveness [4] which generalize among others the prime and search computability of Moschovakis and the Kleene-recursiveness in higher types. In order to get the projective hierarchy of [5] or a hierarchy in the higher types one will have to substitute respective spaces for the space of 1.1 .

\section{Abstract Degrees}

The operation adjunction is used in this section to introduce a degree structure on $F$. 
Lemma 3.9. $a \leqq_{R E} 0$ iff a contains a recursive element.

Lemma 3.10. $\mathrm{dg} \varphi=\mathrm{dg} \varphi^{+}$.

Follows by 1.4 and the equality $\varphi^{+}=\varphi^{++}(I, 0)$.

As a corollary to 3.10 , a $\cap$ Tot $\neq \varnothing$ for all a.

The following statement introduces a jump operator over $D$.

Theorem 3.11. For all a there is a degree a' called the jump of $a$ s.t. $a^{\prime} \leqq_{R E} a$ and whenever $b \leqq_{R E} a$, then $b \leqq a^{\prime}$. Proof: Take a $\varphi \in a$, then take $a^{\prime}=\operatorname{dg} \sum\left(\varphi^{+}\right)$by definition. It is immediate that $a^{\prime} \leqq_{R E} a$. Suppose that $b \leqq_{R E} a$. Then there is a $\psi \in b$ recursive in $\varphi^{+}$, hence $\psi=\bar{m} \Sigma\left(\varphi^{+}\right)$for a certain $\mathrm{m}$. One gets

$$
\psi^{+}=\left(\bar{m} \Sigma\left(\varphi^{+}\right)\right)^{+}=\bar{m} \Sigma\left(\varphi^{+}\right)^{+}(I,[I] R),
$$

hence $\psi \leqq \leqq_{R} \sum\left(\varphi^{+}\right)$, which implies $b \leqq a^{\prime}$. Thereby the proof is completed.

Lemma 3.12. $\quad a<a^{\prime}$. Follows by 1.15 .

Lemma $3.13 . \varliminf_{R E}$ is not antisymmetric.

Proof: $a \leqq_{R E} a^{\prime}$, and $a^{\prime} \leqq_{R E} a$ but $a \neq a^{\prime}$.

Lemma 3.14. $\leqq_{R E}$ is not transitive.

Proof: It is the case that $a^{\prime \prime} \varliminf_{R E} a^{\prime} \varliminf_{R E}$ a. Suppose that $a^{\prime \prime} \leqq_{R E} a$. Then $a^{\prime \prime} \leqq a^{\prime}$ by 3.11 contrary to 3.12 .

Lemma 3.15. $a \leqq b$ implies $a^{\prime} \leqq b^{\prime}$.

Proof: $a^{\prime} \leqq_{R E} a \leqq b$ gives $a^{\prime} \leqq_{R E} b$ by 3.5 , hence $a^{\prime} \leqq b^{\prime}$ by 3.11 . 
REFERENCES

[1] I.I. Ivanov, Iterative operative spaces. Compt.Rend.Acad.Bulg.Sci. 33 (1980), 735-738, in Russian.

[2] I.I. Ivanov, Some examples of iterative operative spaces. Compt.Rend.Acad.Bulg.Sci. 33 (1980), 877-879, in Russian.

[3] I.I. Ivanov, Iterative operative spaces and the system of Scott and de Bakker. Serdica Bulg.Math.Publ. 9 $(1983), 275-288$.

[4] L.I. Ivanov, Axiomatic Recursion Theory - an algebraic approach. To appear.

[5] Y.N. Moschovakis, Abstract first order computability. Trans.Amer.Math.Soc. 138 (1969), 427-504.

[6] Y.N. Moschovakis, on the basic notions in the theory of induction, in: Proceedings of the Fifth International Congress for Logic, Methodology and Philosiphy of Science (Dordrecht, 1977), 207-236. 